\title{
Application of chromatographic analysis for detecting components from polymeric can coatings and further determination in beverage samples
}

\author{
Antía Lestido-Cardama, Patricia Vázquez Loureiro, Raquel Sendón, Perfecto Paseiro Losada, \\ Ana Rodríguez Bernaldo de Quirós*
}

Department of Analytical Chemistry, Nutrition and Food Science. Faculty of Pharmacy, University of Santiago de Compostela, 15782, Santiago de Compostela, Spain.

\section{A R T I C L E I N F O}

\section{Article history:}

Received 26 September 2020

Revised 28 December 2020

Accepted 3 January 2021

Available online 6 January 2021

\section{Keywords:}

HPLC-FLD

Screening

Purge and Trap

Beverage

GC-MS

Exposure

\begin{abstract}
A B S T R A C T
Major type of internal can coating used for food and beverages is made from epoxy resins, which contain among their components bisphenol A (BPA) or bisphenol A diglycidyl ether (BADGE). These components can be released and contaminate the food or beverage. There is no specific European legislation for coatings, but there is legislation on specific substances setting migration limits. Many investigations have paid attention to BPA due to its classification as endocrine disruptor, however, few studies are available concerning to other bisphenol analogues that have been used in the manufacture of these resins.

To evaluate the presence of this family of compounds, ten cans of beverages were taken as study samples. Firstly, the type of coating was verified using an attenuated total reflectance-FTIR spectrometer to check the type of coating presents in most of the samples examined. A screening method was also performed to investigate potential volatiles from polymeric can coatings of beverages using Purge and Trap (P\&T) technique coupled to gas chromatography with mass spectrometry detection (GC-MS).

Moreover, a selective analytical method based on high performance liquid chromatography with fluorescence detection (HPLC-FLD) for the simultaneous identification and quantification of thirteen compounds including bisphenol analogues (BPA, BPB, BPC, BPE, BPF, BPG) and BADGEs (BADGE, BADGE. $\mathrm{H}_{2} \mathrm{O}$, BADGE. $2 \mathrm{H}_{2} \mathrm{O}$, BADGE.HCl, BADGE.2HCl, BADGE.H2O.HCl, cyclo-di-BADGE) in the polymeric can coatings and in the beverage samples was applied. In addition, a liquid chromatography coupled to tandem mass spectrometry (LC-MS/MS) method was optimized for confirmation purposes.

The method showed an adequate linearity $\left(R^{2}>0.9994\right)$ and low detection levels down to $5 \mu \mathrm{g} / \mathrm{L}$. Cyclodi-BADGE was detected in all extracts of polymeric coatings. The concentrations ranged from 0.004 to $0.60 \mathrm{mg} / \mathrm{dm}^{2}$. No detectable amounts of bisphenol related compounds were found in any of the beverage samples at levels that may pose a risk to human health, suggesting a low intake of bisphenols from beverages.
\end{abstract}

(c) 2021 Elsevier B.V. All rights reserved.

\section{Introduction}

Bisphenol A (BPA), 2,2-bis(4-hydroxyphenyl)propane), is the most common bisphenol used primarily as a monomer in the production of polymers, such as polycarbonate plastics and epoxy resins, which are used as a protective coating on the internal

\footnotetext{
* Corresponding author.

E-mail addresses: antia.lestido@usc.es (A. Lestido-Cardama), patriciavazquez.loureiro@usc.es (P. Vázquez Loureiro), raquel.sendon@usc.es (R. Sendón), perfecto.paseiro@usc.es (P. Paseiro Losada), ana.rodriguez.bernaldo@usc.e (A. Rodríguez Bernaldo de Quirós).
}

surface of food and beverage cans to prevent the direct contact. Many of these epoxy resins are synthesised by condensation of BPA with epichlorohydrin to form bisphenol A diglycidyl ether (BADGE). However, when this compound is used in polymer production, residual monomers of BPA remain after incomplete chemical reaction or as results of a chemical degradation or hydrolysis at the ester binding bonds of the polymer. Therefore, this compound may be released and easily migrate into the surrounding medium, such as food and beverages. Its presence in food and beverages is of concern since, with the exception of occupational exposure, it constitutes the main route of human exposure [1]. 
BPA is classified as endocrine disruptor chemical, which are substances whose chemical structure allows them to fit into the binding cavity of the estrogenic receptor influencing the synthesis, transport, secretion, action, binding, or elimination of endogenous hormones in the body and causing adverse health effects such as diabetes, obesity, reproductive disorders, cardiovascular diseases, cancer, changes in behaviour, etc. [1].

Following the recent concern on the use of BPA in food contact material, its use has been reduced lately for those applications. In recent years, it has been reported that residues of other contaminants from the family of bisphenols have been found in canned products. This group of chemical compounds that consist of two phenolic rings bound by either a bridging carbon or other chemical structures, such as bisphenol S (BPS) bisphenol B (BPB), bisphenol $\mathrm{C}$ (BPC), bisphenol E (BPE), bisphenol F (BPF) or bisphenol G (BPG) present physical and chemical properties similar to BPA [1]. However, there is limited information about the safety of these compounds and their possible capability to produce similar or even higher adverse effects than BPA cannot be excluded [2].

The interest on this family of bisphenols relates to their adverse health effects, the enormous production volume, their use in a wide variety of products and objects for consume, as well as their prevalence in the environment [3]. However, there is no specific European legislation for coatings, only there is legislation on specific substances setting migration limits. For example, in 2005, the European Commission fixed a specific migration limit (SML) of 9 $\mathrm{mg} / \mathrm{kg}$ in food or food simulant for BADGE and its hydroxyl derivatives and 1 for its chlorinated derivatives, and also established a tolerable day intake (TDI) of $0.15 \mathrm{mg} / \mathrm{kg}$ of body weight/day for BADGE and its hydrolysis products [4]. In 2015, the European Food Safety Authority (EFSA) re-examined BPA exposure and toxicity issues and established a temporary tolerable daily intake to $4 \mu \mathrm{g} / \mathrm{kg}$ body weight/day [5]. Moreover, recently the European Union Commission lowered the SML for BPA from varnishes or coatings into or onto food to $0.05 \mathrm{mg} / \mathrm{kg}$ of food ( $\mathrm{mg} / \mathrm{kg}$ ), prohibiting the use of BPA in articles intended for infants and young children [6]. However, no migration limits have been established to date for the analogues to BPA. Only, for BPS there is a specific migration limit of $0.05 \mathrm{mg} / \mathrm{kg}$ of food [7].

It has been seen that beverages packaged in cans are more contaminated than those packed in glass, polyethylene terephthalate (PET) or Tetra Pak $[1,8]$. However, in the literature, information on the occurrence of these compounds is scarce and few methods have been described for the analysis of BPA and its analogues in these samples. BPF and BPA were detected in beverage samples at concentration level in the range $0.08-0.68 \mu \mathrm{g} / \mathrm{L}$ [9], and BPB was detected in $50 \%$ of the canned beverages from Portugal tested, with levels ranging from 0.06 to $0.17 \mu \mathrm{g} / \mathrm{L}[10]$.

Since the migration of chemicals from packaging to food and beverages is one of the main concerns of food safety authorities, in this study, a total of ten beverage samples, including alcoholic drinks, energetic drinks, soft drinks and mineral water were investigated. Firstly, the type of coating was verified using an attenuated total reflectance-Fourier transform infrared spectrometer (ATR-FTIR) to check the type of coating presents in the samples examined. Moreover, a screening method was performed to investigate potential volatile susceptible to migrate from polymeric can coatings to beverages. The sample was directly analysed using a Purge and Trap (P\&T) technique, that allows to concentrate the volatiles in a sorbent material, coupled to gas chromatography with mass spectrometry detection (GC-MS).

In the second part of this study, we described a multi-residue method to check the presence of these residual chemicals including BPA, BPB, BPC, BPE, BPF, BPG, BADGE and its hydroxy and chlorinated derivatives (BADGE, BADGE.H2O, BADGE. $\mathrm{H}_{2} \mathrm{O}$, BADGE. $\mathrm{HCl}$, BADGE.2 $\mathrm{HCl}$, BADGE. $\mathrm{H}_{2} \mathrm{O} . \mathrm{HCl}$ ) and cyclo-di-BADGE in the poly- meric can coatings and canned beverages. Determination of all analytes was performed by high-performance liquid chromatography with fluorescence detection (HPLC-FLD) because of the numerous advantages that it offers. This method is sensitive, selective, easy to perform, cheaper than other detection techniques and available in most laboratories [2]. On the contrary, when these compounds are analysed by gas chromatography, a derivatization step is recommended in order to increase their volatility, which requires additional sample manipulation, increase analysis time and reduce the reproducibility [11]. The method developed was validated, evaluating accuracy as mean recoveries, precision in terms of relative standard deviations for within-laboratory reproducibility, as well as the limit of quantification and detection. In addition, a liquid chromatography coupled to tandem mass spectrometry (LC-MS/MS) method was optimized for confirmation purposes of the results obtained.

Finally, the human exposure of bisphenol related compounds associated with this type of beverages was assessed on the bases of measured concentrations and their daily ingestion rates. And the compliance with the European legislation was also checked.

\section{Material and methods}

\subsection{Reagents and standards}

All reagents were analytical grade. Acetonitrile (ACN) HPLC grade and LC-MS grade, methanol (MeOH) HPLC grade and LC-MS grade, butanol for analysis, toluene for analysis and tetrahydrofuran (THF) HPLC grade were provided from Merck (Darmstadt, Germany). Ultrapure water (type I) was obtained from an Autwomatic Plus purification system (Wasserlab, Navarra, Spain).

Analytical standards used for identification: 2,6-di-tert-butyl1,4-benzoquinone $98 \%$, diethyl phthalate $99.5 \%$, benzophenone $99 \%$, caprolactam $99+\%$, octanal $99 \%, \alpha$-pinene $98 \%, \alpha$-terpineol $\geq 90 \%$, hexamethylenetetramine $99 \%$, ethylene glycol butyl ether $\geq 99 \%$, and saturated alkane standard mixture C7-C30 were purchased from Sigma-Aldrich (Schnelldorf, Germany). Triacetin $\geq 99 \%$, 2phenoxyethanol $\geq 99 \%$ and pentanal $\geq 97.5 \%$ were obtained from Fluka (Steinheim, Germany). Nonanal $98.7 \%$ was provided by Supelco (Bellefonte, PA, USA). Phenol $\geq 99.5 \%$ was purchased from Merck (Darmstadt, Germany).

Analytical standards of bisphenols used in the study: bisphenol A (BPA) $\geq 99 \%$ (CAS 80-05-7) was provided by Aldrich-Chemie (Steinheim, Germany). Bisphenol B (BPB) $\geq 98 \%$ (CAS 77-407), bisphenol C (BPC) $\geq 99 \%$ (CAS 79-97-0), bisphenol E (BPE) $\geq 98 \%$ (CAS 2081-08-5), bisphenol F (BPF) $\geq 98 \%$ (CAS 620-92-8), bisphenol G (BPG) $\geq 98 \%$ (CAS 127-54-8), bisphenol A diglycidyl ether (BADGE) $\geq 95 \%$ (CAS 1675-54-3), bisphenol A (3-chloro2-hydroxypropyl) (2,3-dihydroxypropyl) ether (BADGE. $\mathrm{H}_{2} \mathrm{O} \cdot \mathrm{HCl}$ ) $\geq 95 \%$ (CAS 227947-06-0), bisphenol A (3-chloro-2-hydroxypropyl) glycidyl ether (BADGE.HCl) $\geq 90 \%$ (CAS 13836-48-1), and bisphenol A (2,3-dihydroxypropyl) glycidyl ether(BADGE. $\left.\mathrm{H}_{2} \mathrm{O}\right) \geq 95 \%$ (CAS 76002-91-0) were purchased from Sigma-Aldrich (Schnelldorf, Germany). Bisphenol A bis(2,3-dihydroxypropyl) ether (BADGE. $2 \mathrm{H}_{2} \mathrm{O}$ ) $\geq 97 \%$ (CAS 5581-32-8) and bisphenol A bis(3chloro-2-hydroxypropyl) ether (BADGE.2HCl) $\geq 99 \%$ (CAS 480935-2) were obtained from Fluka (Steinheim, Germany). Cyclo-diBADGE 99.5\% (CAS 20583-87-3) was from Chiron AS.

Single stock solutions of individual compounds containing $1000 \mathrm{mg} / \mathrm{L}$ were prepared in acetonitrile, except for the cyclo-diBADGE, for which a solution of $200 \mathrm{mg} / \mathrm{L}$ was prepared in a mixture of ACN:THF (30:20, v/v). A single intermediate mix solution was prepared by dissolving appropriate amounts of all compounds in $90 \% \mathrm{ACN}: \mathrm{H}_{2} \mathrm{O}(\mathrm{v} / \mathrm{v})$ to yield a final concentration of $10 \mathrm{mg} / \mathrm{L}$. Calibration curve was prepared in $45 \%$ ACN using seven concentration standard solutions ranging from 0.0125 to $1 \mathrm{mg} / \mathrm{L}$ and all solutions 
were stored in dark glass bottles in the fridge until the analysis. To avoid BPA contamination, the use of plastics in the laboratory was limited, all the material used was preferably glass. Furthermore, all the glassware had been previously washed with detergent and rinsed with distilled water.

\subsection{Samples and extraction procedure}

A total of ten beverages, including alcoholic drinks (beer, vodka), energetic drinks, soft drinks (tonic, cola) and mineral water were purchased in a local supermarket in Santiago de Compostela (Spain) and were selected as study samples. All of the two-piece cans remained closed and stored at room temperature until the analysis.

To extract the migrants, the cans were opened, emptied and washed with warm water before extraction. A known surface of the internal side of the packaging was put in contact with $100 \mathrm{~mL}$ of acetonitrile for $24 \mathrm{~h}$ in an oven at $70{ }^{\circ} \mathrm{C}$. The can was covered with aluminium foil to avoid evaporation losses. Then, an aliquot of the extract was diluted to half with water type I and filtered through a PTFE $0.22 \mu \mathrm{m}$ filter for HPLC analysis.

To analyse the beverage, part of the content of the can was transferred to a beaker and brought to the ultrasonic bath equipment P-Selecta Ultrasons (Spain) to degas the sample for approximately one hour. The $\mathrm{pH}$ value was measured to verify possible correlation with bisphenol migration into the beverage. Once completely degassed, the sample was extracted according the following method. Briefly, aliquots of $5 \mathrm{~g}$ of each food were taken for analysis. A volume of $5 \mathrm{~mL}$ of heptane solution was added to the sample and stir 1 min using a shaker IKA Vibrax VXR basic (Germany). Then $5 \mathrm{~mL}$ of ACN $90 \%$ were added and stir during $10 \mathrm{~min}$, followed by centrifugation at $1357 \times \mathrm{g}$ for $10 \mathrm{~min}$ at $4{ }^{\circ} \mathrm{C}$ (Hettich Zentrifugen Universal 320R). Finally, the aqueous phase was taken and filtered through a PTFEE $0.22 \mu \mathrm{m}$ filter to be injected in the HPLC. Duplicate tests were performed for each sample.

To perform recovery tests, the sample BC04 was selected, after verifying that it did not present any of the analytes of interest. The recovery was evaluated by spiking the sample at three different concentrations $(0.05,0.1$ and $0.2 \mu \mathrm{g} / \mathrm{g})$ adding $500 \mu \mathrm{L}$ of mixed standard solutions in ACN $90 \%$ and was allowed to infuse into the sample. The spiked samples were extracted in the same way as the samples. Duplicate tests were performed for each level on three consecutive days.

\subsection{Exposure estimation}

Dietary exposure to bisphenol related compounds was estimated taking into account the obtained concentration of the selected analytes in each beverage sample and the Spanish consumption data for each type of beverage obtained from the survey ENALIA 2. According GEMS/Food- EURO recommendations, to estimate dietary exposure, analytical results under the respective limit of detection (LOD) were considered to be equal to one-half of that limit (LOD/2) and values under the limit of quantification (LOQ) were considered to be equal to one-half of that limit (LOQ/2) [12].

ENALIA 2 is a dietary survey conducted in Spain for the adult population between 18 and 74 years of age. It is an individual survey which allows to know the type of food and the quantities consumed (g/day) by this population and the frequency of food consumption, which is essential for scientific research on exposure to other chemical substances through food. The methodology followed the EFSA guidance recommendations on the "General principles for the collection of national food consumption data in the view of a pan-European dietary survey" (EFSA, 2009). The survey included 933 adults and elderly (623 from 18 to 64 years and 310 from 65 to 74 years). In our case, we focus on the adult population group from 18 until 74 years because it is the largest consumer of this type of beverages.

An assessment of the risk associated with dietary exposure was evaluated comparing the obtained chemical intake values with the available TDI values established by authorities like EFSA.

\subsection{Equipment}

\subsubsection{Fourier transform infrared spectroscopy (FTIR)}

To identify the type of polymeric coating, infrared spectra were acquired using an ATR (attenuated total reflectance) - FTIR spectrometer (ATR-PRO-ONE, FTIR 4700, Jasco, Tokyo, Japan) equipped with a diamond optical crystal. This technique allows to examine the samples directly in solid state without requiring additional preparation. The analysis was done on both surfaces (internal and external side) of the lateral and the lid of each sample by covering the entire crystal surface and applying constant and uniform pressure to achieve good spectrum quality. ATR-FTIR spectrometer was controlled by the software Spectra Manager (version 2) in the region from 4000 to $650 \mathrm{~cm}^{-1}$. The spectra identification was performed by using KnowitAll 17.4.135.B software to compare the sample spectra obtained with several commercial database related with polymers (IR Spectral Libraries of Polymers \& Related Compounds from Bio-Rad Laboratories, Inc. Philadelphia, PA, USA). These libraries use algorithms to make decisions about the identity of the material. For this, the hit quality index (HQI) a value that ranges from 0 to 100 (the "best" hit from a search) is calculated in each comparison.

\subsubsection{Gas chromatography (GC)}

For the analysis of potential volatiles from polymeric can coatings a previous step of concentration was performed using a Teledyne Tekmar Stratum Purge and Trap (P\&T) system (Ohio, USA) controlled with the VOC TekLink 3.2 software. The experimental conditions of the P\&T were as follows: Vocarb ${ }^{\mathrm{TM}} 3000$ trap, sample temperature of $90^{\circ} \mathrm{C}$, purge flow of $40 \mathrm{~mL} / \mathrm{min}$, purge time of $20 \mathrm{~min}$, desorb time of $2 \mathrm{~min}$, desorb temperature of $250{ }^{\circ} \mathrm{C}$ and desorb flow of $400 \mathrm{~mL} / \mathrm{min}$.

The GC-MS analysis was carried out using a Finnigan Trace Gas Chromatograph Ultra with a Finnigan Trace DSQ mass detector from Thermo Scientific (California, USA). The volatile compounds were separated on a Rxi-624Sil MS (30 m* $0.25 \mathrm{~mm}$ internal diameter, $1.40 \mu \mathrm{m}$ film thickness) column from Restek (Pennsylvania, USA). The chromatographic conditions were as follows: helium was used as carrier gas at a constant flow rate of $1 \mathrm{~mL} / \mathrm{min}$; the oven program was initially set at $45{ }^{\circ} \mathrm{C}$ for $4 \mathrm{~min}$, then increased at a rate of $8{ }^{\circ} \mathrm{C} / \mathrm{min}$ until $250{ }^{\circ} \mathrm{C}$ and held for $5 \mathrm{~min}$; the transfer line and source temperature were set at $250{ }^{\circ} \mathrm{C}$. The mass spectra were obtained with a mass-selective detector operated under electron impact ionization mode at a voltage of $70 \mathrm{eV}$ and data acquisition was performed in full scan mode over $\mathrm{m} / \mathrm{z}$ range of $20-500$. For data acquisition and processing, Xcalibur 2.0.7 software was used. Compounds were identify using the commercial mass spectral libraries NIST/EPA/NIH 11 (version 2.0) and Wiley RegistryTM 8th edition.

\subsubsection{Liquid chromatography (LC)}

The separation and analysis of bisphenol related compounds, both in extracts and in beverages, was carried out using an analytical method based on high-performed liquid chromatography equipped with a fluorescence detector (HPLC-FLD). Chromatographic measurements were performed with an Agilent Technologies 1200 Series (Waldbronn, Germany) system comprised of a quaternary pump, a degassing device, an autosampler, a column thermostat system, and a fluorescence array detector, all controlled 
by the ChemStation for LC 3D systems software. Fluorescence detection was employed setting $225 \mathrm{~nm}$ as excitation wavelength and $305 \mathrm{~nm}$ as emission wavelength.

Chromatographic conditions were optimized in a previous article of Lestido et al. [13]. Briefly, a Phenosphere $80 \AA$ ODS column (150 mm* $3.2 \mathrm{~mm}$ internal diameter, $3 \mu \mathrm{m}$ particle size) with an appropriate guard column from Phenomenex ${ }^{\circledR}$ (Torrance, CA, USA) was used for the separation of the analytes. The mobile phase consisted of (A) water type I and (B) a mixture of $\mathrm{ACN}: \mathrm{MeOH}$ (50:50, $\mathrm{v} / \mathrm{v}$ ). The gradient elution conditions were: $45 \% \mathrm{~B}$ in an isocratic mode for $2 \mathrm{~min}$, followed by a gradient to $75 \% \mathrm{~B}$ for $14 \mathrm{~min}$, another gradient to $100 \%$ B for 7 min and finally an isocratic elution to $100 \%$ organic phase during $5 \mathrm{~min}$. The delay time for recording the next chromatogram was $5 \mathrm{~min}$. The flow rate was constant at $0.5 \mathrm{~mL} / \mathrm{min}$. The injection volume was $10 \mu \mathrm{L}$. The column oven temperature was kept at $30^{\circ} \mathrm{C}$.

For confirmation of the results, identification of selected compounds was carried out using a high performance liquid chromatography coupled to tandem mass spectrometry (HPLC-MS/MS) system comprised an Accela autosampler, an Accela 1250 pump fitted with a degasser, and a column thermostatized system coupled to a triple stage quadrupole mass spectrometer TSQ Quantum Access max (Thermo Fisher Scientific, San José, CA, USA). Data acquisition and processing were performed using the Xcalibur 2.1.0 software.

The mass spectrometer was operated in positive and negative atmospheric pressure chemical ionisation (APCI) mode. The operating conditions were: nitrogen was used as the sheath gas at a pressure of $35 \mathrm{psi}$, and as auxiliary gas (pressure 10 arbitrary units), argon was used as the collision-induced-dissociation gas in the triple quadrupole instrument at a pressure of $1.0 \mathrm{mTorr}$, the vaporizer temperature and capillary temperature were at $400{ }^{\circ} \mathrm{C}$ and $350{ }^{\circ} \mathrm{C}$, respectively. MS data were acquired in selected reaction monitoring (SRM) mode once the optimization of the MS/MS parameters was performed using the perfusion system. Two transitions of each compound were chosen for identification purposes, and the corresponding collision energy were optimized for maximum intensity. MS/MS conditions with the parent and product ions for bisphenols and BADGEs are described in Lestido et al. [13].

\section{Results and discussion}

\subsection{FTIR Analysis}

As can be seen in Table 1, where the best matches were selected, in general, all samples of beverage cans had polyurethanebased resin on the external lateral, and an internal coating of BADGE-based resin, both on the lateral and on the lid. However, the samples BC05 and BC09 from Germany shown a different composition from the rest. In this case, an internal coating based on acrylic resin was identified on the lateral surface, while in the lid was coated with a phenoxy resin in the external side and polyester in the internal side. Regarding to the external coating on the lateral, it was polypropylene in the sample $\mathrm{BC} 05$ and polyurethanebased resin in the sample BC09.

The FTIR results confirmed that most of the polymeric can coatings used in beverage samples were based on BADGE resins on the inside of the can. The most common epoxy-based coatings are synthesized from bisphenol A and epichlorohydrin forming epoxy resins of bisphenol A diglycidyl ether (BADGE). The success of epoxies as coatings for food cans is due to their desirable flavourretaining characteristics, their excellent chemical resistance and their outstanding mechanical properties [14]. Phenolics are common crosslinkers in epoxide resins and increase their resistance against corrosion and sulphide stains. However, can manufacturers and food industries have begun to innovate and develop alterna-




Table 2

Volatile compounds detected in the non-targeted analysis by P\&T GC-MS.

\begin{tabular}{|c|c|c|c|c|c|c|c|c|c|c|c|c|c|c|c|}
\hline TR & CAS & Name & $\mathrm{m} / \mathrm{z}$ & SI & RSI & BC01 & BC02 & $\mathrm{BCO}$ & BC04 & BC05 & BC06 & $\mathrm{BC} 07$ & BC08 & BC09 & $\mathrm{BC} 10$ \\
\hline 5.69 & $123-72-8$ & Butanal & 44,72 & 836 & 917 & & $\mathrm{X}$ & $\mathrm{X}$ & & $\mathrm{X}$ & $\mathrm{X}$ & $\mathrm{X}$ & $\mathrm{X}$ & $\mathrm{X}$ & \\
\hline 6.90 & $78-83-1$ & Isobutanol & 43,31 & 921 & 921 & & $\mathrm{X}$ & & $\mathrm{X}$ & & $\mathrm{X}$ & & & & $\mathrm{X}$ \\
\hline 7.85 & $71-36-3$ & Butanol* & 56,41 & 943 & 943 & $\mathrm{X}$ & $\mathrm{X}$ & $\mathrm{X}$ & $\mathrm{X}$ & $\mathrm{X}$ & $\mathrm{X}$ & $\mathrm{X}$ & $\mathrm{X}$ & $\mathrm{X}$ & $\mathrm{X}$ \\
\hline 8.36 & $110-62-3$ & Pentanal* & 44,58 & 723 & 830 & & & & & & & & & $\mathrm{X}$ & \\
\hline 9.86 & $108-88-3$ & Toluene* & 91,65 & 789 & 955 & $\mathrm{X}$ & $\mathrm{X}$ & & $\mathrm{X}$ & $\mathrm{X}$ & $\mathrm{X}$ & & $\mathrm{X}$ & $\mathrm{X}$ & \\
\hline 10.48 & $71-41-0$ & Pentanol & $42,55,70$ & 759 & 909 & & & & & & & & & $\mathrm{X}$ & \\
\hline 10.95 & $57-55-6$ & Propylene glycol & 45 & 918 & 950 & & $\mathrm{X}$ & & $\mathrm{X}$ & $\mathrm{X}$ & $\mathrm{X}$ & & & $\mathrm{X}$ & $\mathrm{X}$ \\
\hline 11.07 & $66-25-1$ & Hexanal & 44,56 & 884 & 956 & $\mathrm{X}$ & $\mathrm{X}$ & $\mathrm{X}$ & $\mathrm{X}$ & $\mathrm{X}$ & $\mathrm{X}$ & $\mathrm{X}$ & $\mathrm{X}$ & $\mathrm{X}$ & $\mathrm{X}$ \\
\hline 12.59 & $111-84-2$ & Nonane & $43,57,85$ & 737 & 867 & & & & & & & & $\mathrm{X}$ & & $\mathrm{X}$ \\
\hline 13.51 & $508-32-7$ & Tricyclene & $93,121,136$ & 827 & 879 & & & & & & & & & $\mathrm{X}$ & \\
\hline 13.53 & $111-71-7$ & Heptanal & $70,44,55,81$ & 803 & 900 & & $\mathrm{X}$ & $\mathrm{X}$ & $\mathrm{X}$ & $\mathrm{X}$ & $\mathrm{X}$ & $\mathrm{X}$ & $\mathrm{X}$ & $\mathrm{X}$ & $\mathrm{X}$ \\
\hline 13.75 & $80-56-8$ & $\alpha$-pinene* & 93,77 & 908 & 929 & & & & & $\mathrm{X}$ & & & & & \\
\hline 13.79 & $111-76-2$ & Ethylene glycol butyl ether* & 57,45 & 916 & 922 & $\mathrm{X}$ & $\mathrm{X}$ & $\mathrm{X}$ & $\mathrm{X}$ & $\mathrm{X}$ & $\mathrm{X}$ & $\mathrm{X}$ & $\mathrm{X}$ & $\mathrm{X}$ & $\mathrm{X}$ \\
\hline 14.15 & $471-84-1$ & $\alpha$-Fenchene & $471-84-1$ & 901 & 914 & & & & & & & & & $\mathrm{X}$ & \\
\hline 14.15 & $5131-66-8$ & 2-propanol, 1-butoxy- & $45,57,87$ & 887 & 916 & & & $\mathrm{X}$ & & $\mathrm{X}$ & & & & $\mathrm{X}$ & \\
\hline 14.24 & $79-92-5$ & Camphene & 93,121 & 906 & 930 & & & & & & & & & $\mathrm{X}$ & \\
\hline 14.99 & $123-35-3$ & Myrcene & $69,41,93$ & 921 & 934 & & & & & $\mathrm{X}$ & $\mathrm{X}$ & & & $\mathrm{X}$ & \\
\hline 15.48 & $100-52-7$ & Benzaldehyde & $77,105,51$ & 863 & 960 & $\mathrm{X}$ & $\mathrm{X}$ & & $\mathrm{X}$ & & $\mathrm{X}$ & & $\mathrm{X}$ & & $\mathrm{X}$ \\
\hline 15.52 & 13466-78-9 & 3-carene & $93,77,121$ & 870 & 904 & & & & & $\mathrm{X}$ & & & & & \\
\hline 15.64 & $126-30-7$ & Neopentyl Glycol & 56,73 & 920 & 925 & $\mathrm{X}$ & $\mathrm{X}$ & $\mathrm{X}$ & $\mathrm{X}$ & & $\mathrm{X}$ & $\mathrm{X}$ & $\mathrm{X}$ & $\mathrm{X}$ & $\mathrm{X}$ \\
\hline 15.73 & $142-92-7$ & Hexyl acetate & $43,56,69$ & 851 & 874 & & & & & $\mathrm{X}$ & & & & & \\
\hline 15.76 & $99-86-5$ & $\alpha$-terpinene & $121,93,136$ & 846 & 886 & & & & & & & & & $\mathrm{X}$ & \\
\hline 15.82 & $4719-04-4$ & s-Triazine-1,3,5-triethanol & 86,56 & 720 & 790 & & & & $\mathrm{X}$ & & & & & & \\
\hline 15.92 & $124-13-0$ & Octanal ${ }^{*}$ & $43,56,69$ & 904 & 953 & $\mathrm{X}$ & & $\mathrm{X}$ & $\mathrm{X}$ & & $\mathrm{X}$ & & $\mathrm{X}$ & & \\
\hline 16.04 & $109-52-4$ & Pentanoic acid & 60,73 & 709 & 810 & & & & $\mathrm{X}$ & & & & $\mathrm{X}$ & & \\
\hline 16.08 & $99-87-6$ & p-cymene & 119,134 & 915 & 927 & & $\mathrm{X}$ & & & & $\mathrm{X}$ & $\mathrm{X}$ & & & \\
\hline 16.08 & & Aromatic compound & & & & & & $\mathrm{X}$ & $\mathrm{X}$ & & & $\mathrm{X}$ & $\mathrm{X}$ & $\mathrm{X}$ & $\mathrm{X}$ \\
\hline 16.56 & $104-76-7$ & 1-hexanol, 2-ethyl- & $57,70,83$ & 916 & 921 & & & & & $\mathrm{X}$ & & & & & \\
\hline 16.60 & $99-85-4$ & gamma-terpinene & 93,136 & 919 & 922 & & $\mathrm{X}$ & & & & $\mathrm{X}$ & & & $\mathrm{X}$ & \\
\hline 17.03 & $108-95-2$ & Phenol* & 94,66 & 778 & 901 & $\mathrm{X}$ & $\mathrm{X}$ & $\mathrm{X}$ & $\mathrm{X}$ & & $\mathrm{X}$ & & $\mathrm{X}$ & & $\mathrm{X}$ \\
\hline 17.10 & $1120-21-4$ & Undecane* & $57,43,71$ & 845 & 933 & $\mathrm{X}$ & & $\mathrm{X}$ & & $\mathrm{X}$ & $\mathrm{X}$ & & $\mathrm{X}$ & & \\
\hline 17.22 & $586-62-9$ & Terpinolene & $121,93,136$ & 903 & 919 & & & & & & $\mathrm{X}$ & & & $\mathrm{X}$ & \\
\hline 17.60 & & Aromatic compound & 117,132 & & & & $\mathrm{X}$ & & & $\mathrm{X}$ & $\mathrm{X}$ & & $\mathrm{X}$ & $\mathrm{X}$ & $\mathrm{X}$ \\
\hline 17.95 & $78-70-6$ & Linalool & $71,93,121$ & 925 & 942 & & & & & $\mathrm{X}$ & & & & & \\
\hline 18.09 & $124-19-6$ & Nonanal* & $57,70,82$ & 926 & 944 & $\mathrm{X}$ & $\mathrm{X}$ & $\mathrm{X}$ & $\mathrm{X}$ & $\mathrm{X}$ & $\mathrm{X}$ & $\mathrm{X}$ & $\mathrm{X}$ & $\mathrm{X}$ & $\mathrm{X}$ \\
\hline 18.21 & $1632-70-8$ & 5-methyl-undecane & $71,57,85$ & 890 & 894 & & & & & $\mathrm{X}$ & & & & & \\
\hline 18.55 & $1002-43-3$ & 3-methyl-undecane & $57,71,85$ & 824 & 837 & & & & & $\mathrm{X}$ & & & & & \\
\hline 18.60 & $103-09-3$ & 2-Ethylhexylacetate & $70,83,43$ & 896 & 903 & & & & & $\mathrm{X}$ & & & & & \\
\hline 18.76 & $1632-73-1$ & Fenchol & 81,107 & 881 & 910 & & & & & & & & & $\mathrm{X}$ & \\
\hline 18.84 & $586-82-3$ & 1-terpineol & $81,121,136$ & 867 & 876 & & & & & & & & & $\mathrm{X}$ & \\
\hline 19.11 & $112-40-3$ & Dodecane & $57,71,85$ & 912 & 912 & $\mathrm{X}$ & $\mathrm{X}$ & $\mathrm{X}$ & $\mathrm{X}$ & $\mathrm{X}$ & $\mathrm{X}$ & & $\mathrm{X}$ & $\mathrm{X}$ & $\mathrm{X}$ \\
\hline 19.23 & $7299-40-3$ & $\beta$-terpineol & $71,93,136$ & 910 & 911 & & & & & & & & & $\mathrm{X}$ & \\
\hline 19.50 & $106-32-1$ & Ethyl octanoate & $88,101,127$ & 858 & 902 & $\mathrm{X}$ & $\mathrm{X}$ & $\mathrm{X}$ & & & & & & & \\
\hline 19.57 & $93-89-0$ & Ethylbenzoate & $105,77,122$ & 706 & 879 & & $\mathrm{X}$ & & & & & & & & \\
\hline 19.72 & $562-74-3$ & 4-terpineol & 71,111 & 876 & 907 & & & & & & & & & $\mathrm{X}$ & \\
\hline 19.97 & $112-34-5$ & ethanol, 2-(2-butoxyethoxy)- & $57,45,41$ & 752 & 874 & & $\mathrm{X}$ & & & & & & & & $\mathrm{X}$ \\
\hline 20.07 & $112-31-2$ & Decanal & $57,71,82$ & 929 & 938 & $\mathrm{X}$ & & $\mathrm{X}$ & $\mathrm{X}$ & & $\mathrm{X}$ & $\mathrm{X}$ & $\mathrm{X}$ & & $\mathrm{X}$ \\
\hline 20.09 & $98-55-5$ & $\alpha$-terpineol* & $59,93,121$ & 914 & 921 & & & & & $\mathrm{X}$ & & & & $\mathrm{X}$ & \\
\hline 20.22 & $119-36-8$ & Methyl salicylate & $120,92,152$ & 817 & 845 & & & & $\mathrm{X}$ & & & & & & \\
\hline 20.38 & $502-44-3$ & 2-oxepanone & $55,42,84$ & 875 & 884 & & & & $\mathrm{X}$ & & $\mathrm{X}$ & & & & \\
\hline 20.97 & $629-50-5$ & Tridecane & $42,57,71$ & 766 & 859 & & & $\mathrm{X}$ & & $\mathrm{X}$ & & & $\mathrm{X}$ & & $\mathrm{X}$ \\
\hline 20.97 & $100-97-0$ & Hexamethylenetetramine* & 42,140 & 907 & 930 & $\mathrm{X}$ & & & $\mathrm{X}$ & & $\mathrm{X}$ & & & & \\
\hline 21.16 & $122-99-6$ & 2-phenoxyethanol* & $94,77,138$ & 734 & 841 & & & & $\mathrm{X}$ & & & & & & \\
\hline 21.32 & $99-49-0$ & Carvone & $82,108,54$ & 918 & 945 & & & & & $\mathrm{X}$ & $\mathrm{X}$ & & & $\mathrm{X}$ & \\
\hline 22.36 & $89-83-8$ & Thymol & 135,150 & 901 & 916 & & & & & & & & & $\mathrm{X}$ & \\
\hline 22.47 & $105-60-2$ & Caprolactam* & $56,113,85$ & 824 & 971 & & $\mathrm{X}$ & $\mathrm{X}$ & & & & $\mathrm{X}$ & $\mathrm{X}$ & & $\mathrm{X}$ \\
\hline 22.66 & $499-75-2$ & Carvacrol & 135,150 & 765 & 848 & & & & & & & & & $\mathrm{X}$ & \\
\hline 22.74 & $629-59-4$ & Tetradecane* & $57,71,85$ & 747 & 896 & & & $\mathrm{X}$ & $\mathrm{X}$ & $\mathrm{X}$ & & & $\mathrm{X}$ & $\mathrm{X}$ & $\mathrm{X}$ \\
\hline 22.94 & $6378-65-0$ & Hexyl hexanoate & $43,117,56$ & 844 & 882 & & & & & $\mathrm{X}$ & & & & & \\
\hline 22.94 & $102-76-1$ & Triacetin* & $43,145,103$ & 739 & 911 & & $\mathrm{X}$ & $\mathrm{X}$ & $\mathrm{X}$ & & $\mathrm{X}$ & $\mathrm{X}$ & & & $\mathrm{X}$ \\
\hline 23.23 & $515-13-9$ & Cyclohexane & $81,93,107$ & 853 & 881 & & & & & & & & & $\mathrm{X}$ & \\
\hline 23.69 & $112-54-9$ & Dodecanal & $57,69,82$ & 706 & 861 & & & & & & & & $\mathrm{X}$ & & $\mathrm{X}$ \\
\hline 25.03 & $719-22-2$ & 2,6-di-tert-butyl-1,4-benzoquinone* & $177,135,220$ & 840 & 871 & & $\mathrm{X}$ & $\mathrm{X}$ & & & $\mathrm{X}$ & & $\mathrm{X}$ & & $\mathrm{X}$ \\
\hline 25.26 & $483-76-1$ & d-Cadinene & $161,119,204$ & 906 & 911 & & & & & & & & & $\mathrm{X}$ & \\
\hline 25.32 & 20307-83-9 & $\beta$-sesquiphellandrene & $69,92,133$ & 785 & 816 & & & & & & & & & $\mathrm{X}$ & \\
\hline 26.44 & & Glutaric acid compound & 115 & 757 & 868 & & $\mathrm{X}$ & $\mathrm{X}$ & & & & $\mathrm{X}$ & & & $\mathrm{X}$ \\
\hline 26.83 & & Cyclohexanecarboxylic acid compound & & & & & & & & & $\mathrm{X}$ & & $\mathrm{X}$ & & \\
\hline 27.23 & $84-66-2$ & Diethyl phthalate* & 149,177 & 925 & 939 & $\mathrm{X}$ & $\mathrm{X}$ & $\mathrm{X}$ & $\mathrm{X}$ & $\mathrm{X}$ & $\mathrm{X}$ & $\mathrm{X}$ & $\mathrm{X}$ & $\mathrm{X}$ & $\mathrm{X}$ \\
\hline 28.00 & $119-61-9$ & Benzophenone* & $105,77,182$ & 704 & 929 & & & & & & $\mathrm{X}$ & & & & \\
\hline 29.06 & $481-34-5$ & Cardinol & $43,95,121$ & 720 & 859 & & & & & & & & & $\mathrm{X}$ & \\
\hline 30.03 & & Cyclopentanecarboxylic acid compound & $115,69,97$ & & & $\mathrm{X}$ & $\mathrm{X}$ & $\mathrm{X}$ & $\mathrm{X}$ & & $\mathrm{X}$ & & & & \\
\hline
\end{tabular}

$*$ : confirmed with standards. 


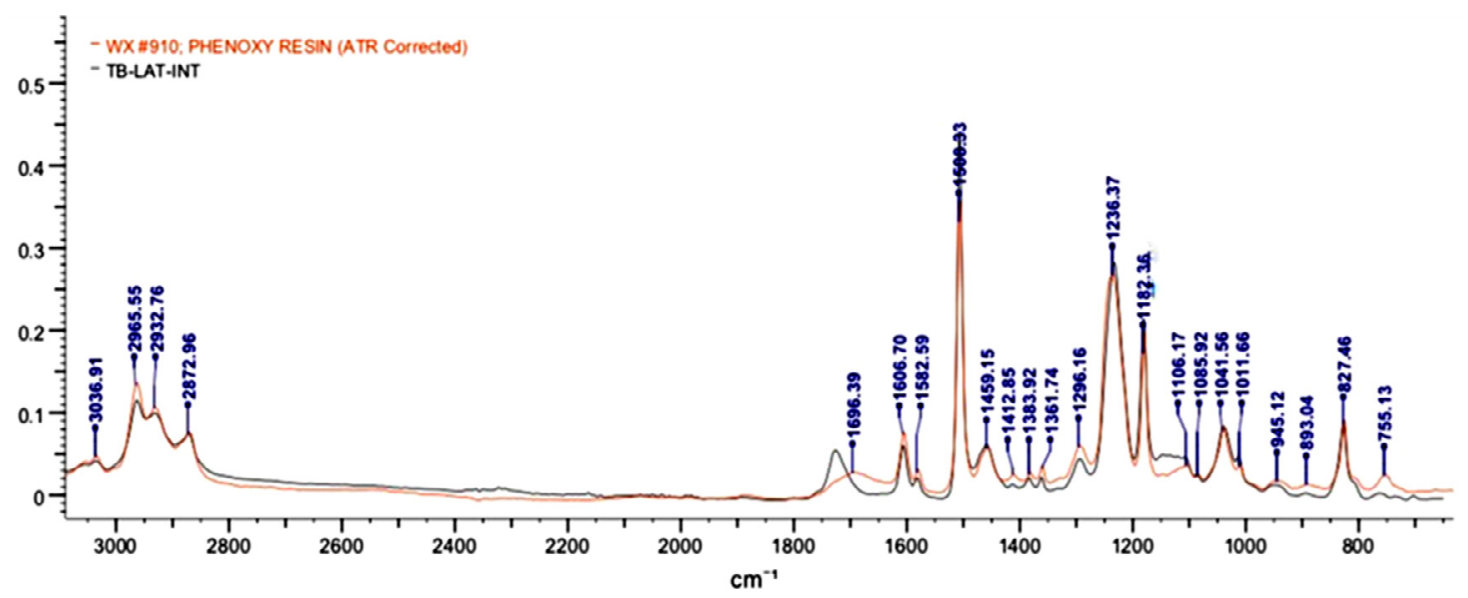

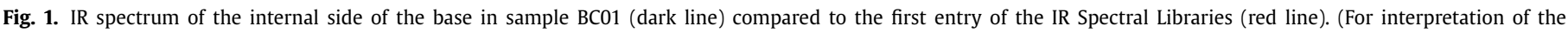
references to color in this figure legend, the reader is referred to the web version of this article.)

tives to replace food contact materials based on BPA epoxy resins as a consequence of the uncertainty of the toxic effects reported, public discussions, and recent regulatory decisions. Acrylic resins and polyester coatings are currently in use as first-generation alternatives [15].

Polyurethanes are a polymeric material with numerous applications in the coating industries due to their good properties such as mechanical strength, abrasion resistance, toughness, low temperature flexibility, chemical and corrosion resistance. These polymeric plasticizers are incorporated in the ink formulation of the packaging to provide a non-migrating character, improve adhesion and resistance to water and deep freeze [16].

Fig. 1 shown the spectrum corresponding to the internal lateral of the beer sample BC01 (black line) overlaid with the first entry of the IR Spectral Libraries (red line). The main material identified was an epoxy resin coating with an HQI of 96.70. This assignment is carried out by the identification of the different chemical groups that make up the spectrum.

\subsection{Screening of volatile compounds in cans}

A total of 71 volatile compounds were detected in the nontargeted analysis of the ten samples of cans (Table 2). Eighteen compounds could be positively confirmed by injection of the respective standard comparing the retention times and their respective mass spectra, and the rest of the peaks were tentatively identified by comparison of the mass spectra with the library entries. Only compounds with the best direct matching factors (SI) and reverse search matching (RSI) found during the library search were considered for the study. Fig. 2 show the GC-MS chromatogram of the sample BC08. As can be seen, the most intense peak corresponds to limonene, probably it comes to the beverage.

It is important to consider that, in this study, the samples analysed were already in contact with the food, since the material was not available prior to contact. Therefore, the mass transfer could take place in both directions, migration from the packaging to the food and sorption from the food into the packaging. Moreover, it should be taken into account that the analysis of the material includes both sides, internal and external.

Any bisphenol related compounds were not identified by GCMS at low concentrations due to their low volatility. However, a wide variety of compounds including alkanes (nonane, undecane, dodecane, tridecane, tetradecane), alcohols (butanol, isobutanol, pentanol), and aldehydes (butanal, pentanal, hexanal, heptanal, octanal, nonanal, decanal, dodecanal, tetradecanal) were identified.
Some epoxy resins could be cured (cross-linked and modified) by phenolic resins that consist of oligomeric materials prepared from phenol, formaldehyde and butanol [17]. It could be why phenol was detected in several samples (BC01, BC02, BC03, BCO4, $\mathrm{BC} 06, \mathrm{BC} 08, \mathrm{BC} 10)$, while its homologues such as thymol and its isomer carvacrol were found in sample BC09 [18]. The formaldehyde releaser triazine-triethanol, which is used as cooling agent for metal processing, lubricant, paint, lacquers and varnishes or printing inks was detected in sample BC04 [19]. Hexamethylenetetramine, an epoxy hardener, was identified in samples BC01, BC04 and BC06 [20].

Neopentyl glycol and propylene glycol, which are often used as intermediate substances in the production of polyester resins and polyurethanes [21,22], were found in several samples. 2Oxepanone, detected in samples BC04 and BC06, is used for the modification of acrylic resins and polyesters, but it is also used for modifying epoxy resins and polyurethanes [23]. It was detected as a print-related contaminant in food packaging by Lago et al. [24]. The compounds 1-hexanol-2-ethyl and 2-ethylhexylacetate, which were identified in sample BC05, could be impurities from the commercial 2-ethylhexylacrylate, a monomer used in the production of acrylic adhesives [25]. These results are in accordance with the FTIR-ATR coating type identification.

Printing inks used in food packaging materials usually consist of colouring matters (pigments or dyes), vehicles (resins), solvents and a large number of additives, such as plasticisers or UV absorbers, that improve the properties of printing inks [26]. Some solvents that are used in coating formulations were identified in our samples such as cyclohexane, toluene, 2-ethyl hexanol and ethylene glycol butyl ether [27]. Hexyl acetate, identified in sample BC05, is employed as adhesive and plasticizer [28]. Methyl salicylate, which was found in sample BCO4, is used as a UV-light stabilizer [29]. Ethylbenzoate, which is used as a solvent or can be a reaction by-product from UV-printing, was detected in sample BC02. Benzophenone, identified in sample BC06, is a photoinitiator for UV-inks. Caprolactam was present in the external colour printings of the samples $\mathrm{BC} 02, \mathrm{BC} 03, \mathrm{BC} 07, \mathrm{BC} 08, \mathrm{BC} 10$ [30]. Triacetin, among its applications, is used on printing inks applied to the non-food contact surface of food packaging materials and articles and was identified in six samples (BC02, BC03, BC04, $\mathrm{BC} 06, \mathrm{BC} 07, \mathrm{BC} 10)$ [31]. Other chemical compounds related with inks detected in tested samples were 2-(2-butoxyethoxy)-ethanol (BC02, BC10) [30], 2-phenoxyethanol (BC04) [32] and 1-butoxy-2propanol (BC03, BC05, BC09) [33]. Diethyl phthalate, a plasticizer widely used in resins, polymers, adhesives, paints and lacquers, 


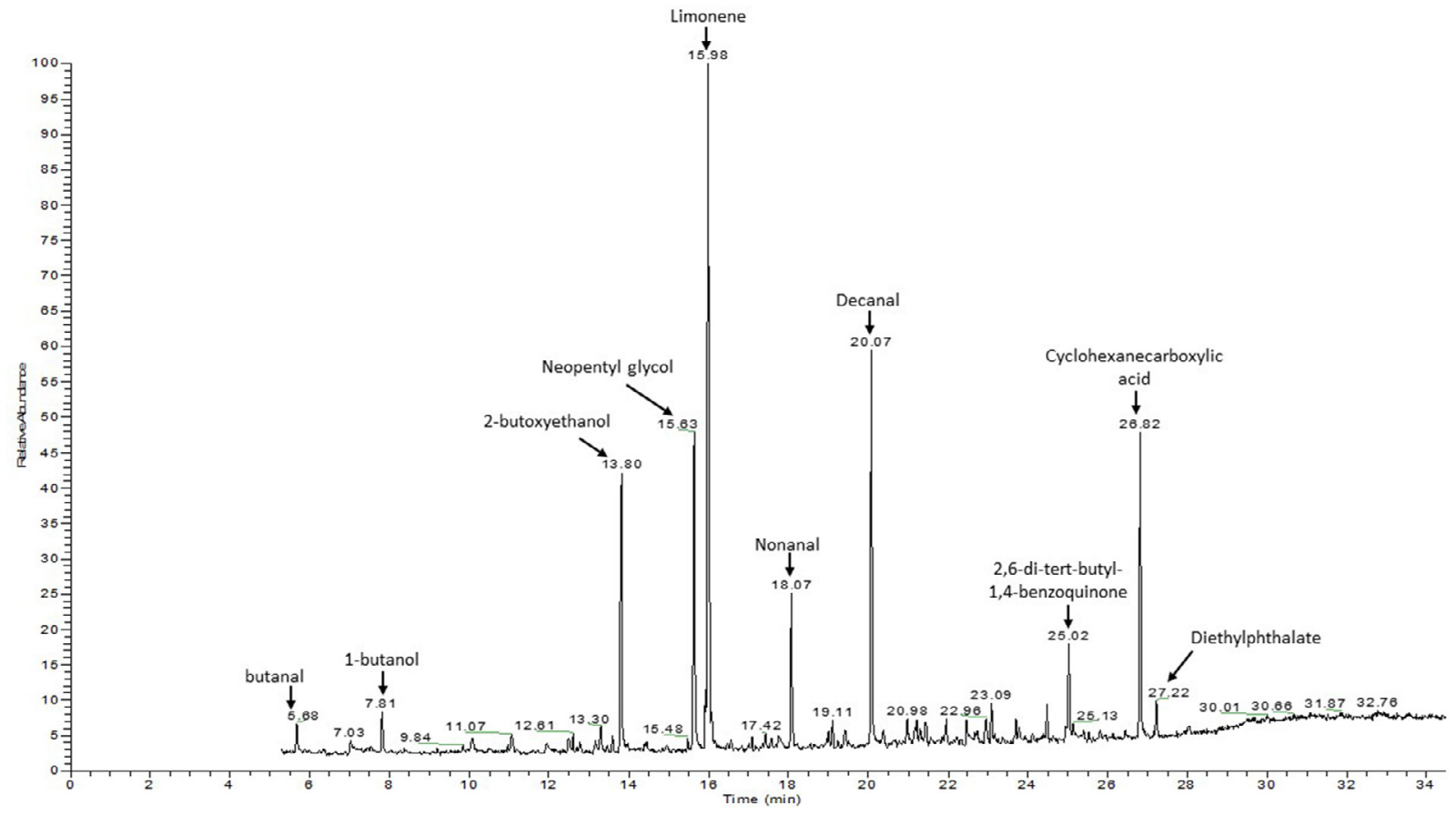

Fig. 2. GC-MS chromatogram of the polymeric can coating in sample BC08 with the identification of some peaks.

was found in all the polymeric can coatings analysed. It is interesting to note that this compound has been reported in alcoholic drinks and soft drinks as described by Russo et al. [34].

2,6-di-tert-butyl-1,4-benzoquinone, a well-known degradation product from antioxidant additives type Irganox and Irgafos was detected in five samples (BC02, BC03, BC06, BC08, BC10) [13,30.

In our analyses, a series of compounds from the family of terpenes including $\alpha$-pinene, 3-carene, camphene, myrcene, $\alpha$-terpinene, gamma-terpinene, tricyclene, p-cymene, carvone, d-cadinene, $\beta$-sesquiphellandrene, cardinol, carvacrol, fenchol, $\alpha$-fenchene, linalool, terpinolene, 4-terpineol, $\alpha$-terpineol, $\beta$ terpineol and 1-terpineol were found in samples BC05, BC06 and BC09. This type of compounds generally are used as flavourings although, however, other studies have reported the use of terpenebased resins for many years in commercial applications such as adhesives, printing inks, coatings and tackifiers [35].

Some other compounds such as benzaldehyde (BC01, BC02, $\mathrm{BC} 04, \mathrm{BC06}, \mathrm{BC08}, \mathrm{BC} 10)$, hexyl hexanoate (BC05), 5-methylundecane (BC05) has also been found in packaging materials as reported by Nerín et al. [36,37], on the other hand, 3-methylundecane (BC05) was detected in recycled high-density polyethylene [38].

There is another similar work carried out by Bradley et al. [39] where volatile potential migrants in the epoxy phenolic coating were determined by headspace GC-MS. However, in the present study, P\&T system was used in order to concentrate the sample as an additional step.

\subsection{Analysis of polymeric can coatings}

Table 3 presents a summary of the bisphenol related compounds identified in the extracts of the polymeric can coating and their concentration obtained by HPLC-FLD. The identification of the analytes in the acetonitrile extracts was based on the comparison of the fluorescence spectra and retention times with those obtained by analysing, under the same conditions, a mix standard solution containing the analytes of interest. The analysis of each extract was carried out in duplicate. Furthermore, to ensure that contamination was minimal, blanks were injected into the sequence.

The quantification was performed by external calibration curve method. A series of standard solutions of known concentration were analysed during each working session to test the linearity of the method. Calibration curves were constructed representing the chromatographic peaks area against standard solution concentration. In the case of cyclo-di-BADGE, the quantification was carried out as the sum of the two isomers. All of them have shown good linearity in the concentration range with determination coefficients $\left(r^{2}\right) \geq 0.9994$. The repeatability within day was determined by analysing ten replicates of the standards at a concentration level of $0.025 \mathrm{mg} / \mathrm{L}$, expressed as the percentage of RSD $(n=10)$ was always lower than $5 \%$ for all the analytes. The areas of the samples obtained by HPLC-FLD were interpolated in the calibration curve of each compound obtaining the concentrations reported in Table 3.

As was reported in the article of Lestido et al. [13], the limits of detection (LOD) defined as signal three times the height of the noise level, and quantification (LOQ) defined as signal ten times the height of the noise level (corresponding to the lowest calibration level of the calibration curve) achieved with this method by HPLC-FLD were $0.005 \mathrm{mg} / \mathrm{L}$ and $0.0125 \mathrm{mg} / \mathrm{L}$, respectively. So, the method shows enough sensitivity to detect the analytes at the regulatory levels required.

To confirm the identity of the analytes detected in the samples, the transition reactions monitored by LC-MS/MS and the retention times of these ions were compared with those obtained when analysing, under the same conditions, a mix standard solution of the analytes of interest. In the case of the LC-MS/MS developed method, the sensitivity was evaluated on limits of detection (LOD), which was estimated as the lowest concentration that provided a signal-to-noise ratio $(\mathrm{S} / \mathrm{N})$ higher than three for both transitions. The method shows a good sensitivity with LODs of $0.5 \mu \mathrm{g} / \mathrm{L}$ for cyclo-di-BADGE; $1 \mu \mathrm{g} / \mathrm{L}$ for BPE, BPG and BADGE; $5 \mu \mathrm{g} / \mathrm{L}$ for BPF, BPA, BPB, BPC, BADGE. $2 \mathrm{H}_{2} \mathrm{O}$, BADGE. $\mathrm{H}_{2} \mathrm{O}$ and BADGE. $\mathrm{HCl}$; and $0.5 \mu \mathrm{g} / \mathrm{mL}$ for BADGE. $2 \mathrm{HCl}$ and BADGE. $\mathrm{H}_{2} \mathrm{O} . \mathrm{HCl}$.

Among all the bisphenol analogues analysed, only levels of BPA above the detection limit was detected in 4 samples (BC03, BCO4, 
Table 3

Bisphenol related compounds identified in the extracts of the analysed cans and their concentrations $\left(\mathrm{mg} / \mathrm{dm}^{2}\right) \mathrm{by}$ HPLC-FLD.

\begin{tabular}{lllllllllll}
\hline & BC01 & BC02 & BC03 & BC04 & BC05 & BC06 & BC07 & BC08 & BC09 & BC10 \\
\hline BPF & - & - & - & - & - & - & - & - & - & - \\
BADGE.2H $H_{2} \mathrm{O}$ & 0.002 & - & 0.004 & 0.003 & - & 0.002 & 0.006 & 0.004 & - & 0.004 \\
BPE & - & - & - & - & - & - & - & - & - & - \\
BPA & - & - & 0.003 & 0.003 & - & - & 0.003 & - & - & 0.003 \\
BPB & - & - & - & - & - & - & - & - & - & - \\
BADGE. $\mathrm{H}_{2} \mathrm{O}$ & - & - & - & - & - & - & - & - & - & - \\
BADGE. $\mathrm{H}_{2} \mathrm{O} . \mathrm{HCl}$ & - & - & - & $<$ LOQ & - & - & $<$ LOQ & - & - & - \\
BPC & - & - & - & - & - & - & - & - & - & - \\
BADGE & - & - & - & - & - & - & $<$ LOQ & - & - & - \\
BADGE.HCl & - & - & - & - & - & - & - & - & - & - \\
BADGE.2HCl & - & - & - & - & - & - & - & - & - & - \\
BPG & - & - & - & - & - & - & - & - & - & - \\
Ciclo-di-BADGE & 0.26 & 0.17 & 0.36 & 0.43 & 0.006 & 0.37 & 0.60 & 0.40 & 0.004 & 0.30 \\
\hline
\end{tabular}

* LOQ: limit of quantification considering the signal by LC-MS/MS

BC07 and BC10) with an average concentration of $0.003 \mathrm{mg} / \mathrm{dm}^{2}$, which is well below the SML established. Among the BADGE derivatives, BADGE. $2 \mathrm{H}_{2} \mathrm{O}$ was detected in seven samples at an average concentration of $0.004 \mathrm{mg} / \mathrm{dm}^{2}$. This fact supports the theory that BADGE is unstable in water-based food because it can be hydrolyse, and the hydrolyses derivatives may be the best markers for exposure to these compound [40]. Cyclo-di-BADGE was detected in all samples analysed in the concentration range of 0.004$0.60 \mathrm{mg} / \mathrm{dm}^{2}$. Fig. 3 shows an example of a chromatogram where the two isomers of cyclo-di-BADGE can be seen in an aliquot of the sample BC04.

As can be seen from the data obtained, the lower levels of the analytes were found in samples $\mathrm{BC} 02, \mathrm{BC} 05$ and $\mathrm{BC} 09$. In the case of the samples BC05 and BC09 from Germany, although their internal coatings were identified as acrylic resin in the lateral and polyester in the lid, and should not contain bisphenols, low levels of cyclo-di-BADGE were detected by both techniques. Some scientific research articles have reported the presence of bisphenols in various foodstuffs, even when the chemical nature of their packaging should not allow their release [2]. This migration could take place due to possible set-off phenomena described during the manufacturing process and storage of packaging materials in the industry [41].

\subsection{Analysis of beverage samples}

The chromatographic parameters used in the HPLC method were found to be optimal in order to identify and quantify the analytes in a complex matrix such as a beer sample. Method performance was evaluated by spiking experiments carried out at three different levels $(0.05,0.1$ and $0.2 \mu \mathrm{g} / \mathrm{g})$ during three different days. Fortified samples were analysed in duplicate and quantified. For each spiking level and all compounds, method accuracy was calculated in terms of mean percentage recoveries, and precision, as relative standard deviation (RSD). The recovery was calculated by comparing the theoretical concentration spiked and the concentration value obtained from HPLC. Recoveries $(n=6)$ were in the range $75-102 \%$ and the RSD was less than $10 \%$ in all the cases as can be seen in Table 4.

The results of the analysis carried out on the beverages by HPLC-FLD were negative on all occasions, that means, no analytes were detected above the detection limit in any of the samples. The LC-MS/MS analysis confirmed these results.

These results are in line with those reported by other authors if we take into account the sensitivity of our methods. For example, just like our results, Rozaini et al. [42] not detected BPA in any of the beverage samples analysed by HPLC-DAD, but neither does it

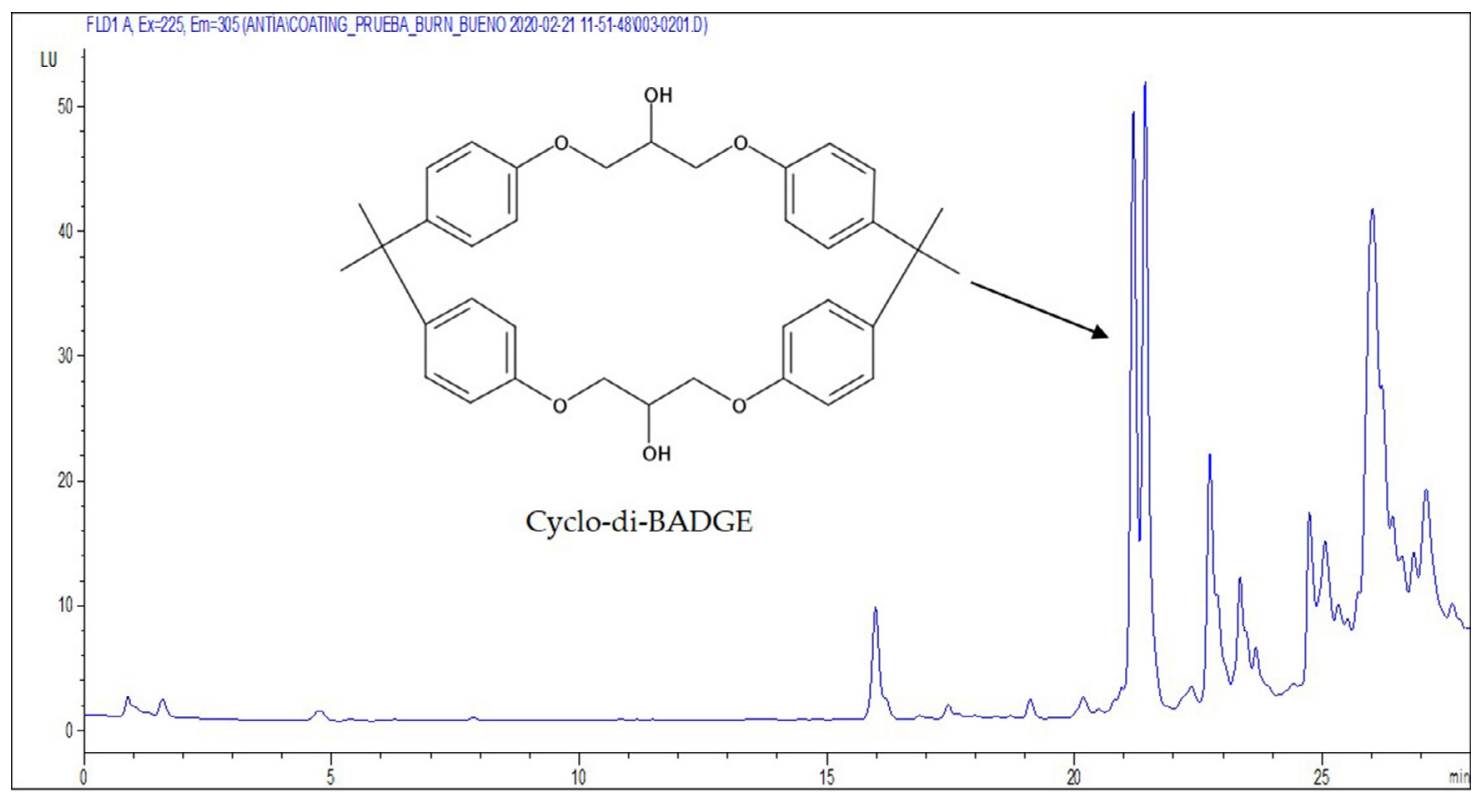

Fig. 3. HPLC-FLD chromatogram of an extract of the can sample BCO4 and the chemical structure of the cyclo-di-BADGE. 
Table 4

\begin{tabular}{|c|c|c|c|c|c|c|}
\hline \multirow[t]{2}{*}{ Compound } & \multicolumn{3}{|c|}{ Recovery (\%) $(n=6)$} & \multicolumn{3}{|c|}{ Intermediate Precision (RSD $\%)(n=6)$} \\
\hline & $0.05 \mu \mathrm{g} / \mathrm{g}$ & $0.1 \mu \mathrm{g} / \mathrm{g}$ & $0.2 \mu \mathrm{g} / \mathrm{g}$ & $0.05 \mu \mathrm{g} / \mathrm{g}$ & $0.1 \mu \mathrm{g} / \mathrm{g}$ & $0.2 \mu \mathrm{g} / \mathrm{g}$ \\
\hline $\mathrm{BPF}$ & 88 & 88 & 96 & 6 & 6 & 3 \\
\hline BADGE. $2 \mathrm{H}_{2} \mathrm{O}$ & 83 & 83 & 96 & 3 & 3 & 3 \\
\hline $\mathrm{BPE}$ & 92 & 89 & 102 & 7 & 9 & 8 \\
\hline BPA & 87 & 91 & 99 & 3 & 5 & 8 \\
\hline BPB & 84 & 89 & 95 & 5 & 4 & 3 \\
\hline BADGE. $\mathrm{H}_{2} \mathrm{O}$ & 78 & 81 & 91 & 4 & 3 & 3 \\
\hline BADGE. $\mathrm{H}_{2} \mathrm{O} \cdot \mathrm{HCl}$ & 87 & 84 & 96 & 7 & 5 & 4 \\
\hline $\mathrm{BPC}$ & 86 & 85 & 99 & 7 & 6 & 3 \\
\hline BADGE & 80 & 82 & 87 & 4 & 3 & 6 \\
\hline BADGE.HCl & 85 & 90 & 93 & 1 & 3 & 5 \\
\hline BADGE.2HCl & 83 & 86 & 96 & 5 & 2 & 6 \\
\hline BPG & 82 & 86 & 97 & 3 & 5 & 6 \\
\hline CYDBADGE & 79 & 91 & 75 & 2 & 10 & 3 \\
\hline
\end{tabular}

specify in their study the type of packaging in which these samples were. On the contrary, in the study conducted by Gallo et al. [1], 40 energy drinks were analysed by UPLC-FLD and the highest concentrations quantified for BPA $(3.3 \mathrm{ng} / \mathrm{mL})$ and BPF $(1.3 \mathrm{ng} / \mathrm{mL})$ were below our detection limit of $0.005 \mathrm{mg} / \mathrm{L}$, while BPB was no detect in any of the samples. However, BADGE was detected at value above our limit of quantification in two samples with concentrations of 13.4 and $19.4 \mathrm{ng} / \mathrm{mL}$ [1]. In the study carried out by Yang et al. [8], BPA and BPF were the most frequently detected bisphenols in the beverage samples and the concentrations obtained varied from not detected to $12 \mathrm{ng} / \mathrm{mL}$ and $0.39 \mathrm{ng} / \mathrm{mL}$, respectively, while BPB was not detected in any of the samples. In another work, Gallart-Ayala et al. [11] analysed carbonated beverage from Spain and reported values for BPA and BPF of $607 \mathrm{ng} / \mathrm{L}$ and $218 \mathrm{ng} / \mathrm{L}$, respectively, while $\mathrm{BPB}$ and $\mathrm{BPE}$ were no detect in any of the samples.

\subsection{Estimation of the dietary exposure}

According GEMS/Food- EURO recommendations, to estimate dietary exposure, analytical results under the respective LOD were considered to be equal to one-half of that limit (LOD/2) [12]. Low dietary exposure data to this type of analytes were found in the samples of beverages analysed in this study. The mean dietary exposure was in the range of $0.01-0.02 \mu \mathrm{g} / \mathrm{kg}$ bw per day. The highest estimated dietary exposure in the 95th percentile was $0.05 \mu \mathrm{g} / \mathrm{kg}$ bw per day for all the analytes in the sample BC10, which correspond to natural mineral water drink due to its high consumption.

From the public health standpoint, it is interesting to note that in all the samples of beverages analysed in the study, BADGE and its hydrolysis products exposure turned out to be lower than their established TDI of 0.15 of bw/day [4], and BPA was far below its t-TDI of $4 \mu \mathrm{g} / \mathrm{kg}$ of bw/day [5], which demonstrate the safety of the studied coatings present in the market. Regarding to the other analogues of bisphenols analysed and cyclo-di-BADGE, this comparison was not possible, because international organizations have not set regulations on their presence in food and beverages, migration limits or TDI values [43]. In this case, when no toxicity data are available, the threshold of toxicological concern (TTC) based Cramer structural class can be a useful tool for its evaluation [44]. Thus, cyclo-di-BADGE, BPF, BPE, BPB and BPC that are classified as III class according Cramer rules, present a threshold of $1.5 \mu \mathrm{g} / \mathrm{kg}$ bw/day [44], which is three times above the value obtained in the 95th percentile for the sample BC10.

However, the concern about possible cocktail effects due to the joint contamination by bisphenol related compounds has not yet addressed and needs to be taken into account for risk assessment [1].

\section{Conclusion}

A non-target analysis by P\&T GC-MS allowed the identification of 71 volatile compounds, proving that it is a powerful tool for screening purposes and determine the components used in the formulation of coatings. To the best of our knowledge very limited literature about the application of this technique to analyse polymeric coatings have been reported.

A multi-residue method based on HPLC-FLD was employed to identify and quantify thirteen bisphenol related compounds in the polymeric can coatings and their beverage samples, appropriate linearity, accuracy, and precision was achieved. The positive confirmation of the results was carried out using liquid chromatography with tandem mass spectrometry (LC-MS/MS).

Most of the analysed samples had an internal epoxy-phenoxy resin coating. In the extracts from the can coatings BPA, BADGE, BADGE. $2 \mathrm{H}_{2} \mathrm{O}$, BADGE. $\mathrm{H}_{2} \mathrm{O} . \mathrm{HCl}$ and cyclo-di-BADGE were detected and concentrations below LOD for all analytes were found in the beverage samples.

From the food safety point of view, it can be concluded that they comply with the European legislation, suggesting a low intake of bisphenols from beverages.

\section{Funding}

The study was financially supported by the Ministerio de Ciencia, Innovación y Universidades, by Fondo Europeo de Desarrollo Regional (FEDER), and by Agencia Estatal de Investigación Ref. No. PGC2018-094518-B-I00 “MIGRACOATING” (MINECO/FEDER, UE).

\section{Declaration of Competing Interest}

The authors declare that they have no known competing financial interests or personal relationships that could have appeared to influence the work reported in this paper.

\section{CRediT authorship contribution statement}

Antía Lestido-Cardama: Methodology, Software, Investigation, Writing - original draft. Patricia Vázquez Loureiro: Methodology, Software. Raquel Sendón: Conceptualization, Writing - review \& editing, Supervision, Project administration. Perfecto Paseiro Losada: Conceptualization, Writing - review \& editing, Supervision. Ana Rodríguez Bernaldo de Quirós: Conceptualization, Writing review \& editing, Supervision, Project administration, Funding acquisition. 


\section{Acknowledgments}

Antía Lestido is grateful for her grant "Programa de axudas á etapa predoutoral" da Xunta de Galicia (Consellería de Cultura, Educación e Ordenación Universitaria).

\section{References}

[1] P. Gallo, I.D.M. Pisciottano, F. Esposito, E. Fasano, G. Scognamiglio, G.D. Mita, T Cirillo, Determination of BPA, BPB, BPF, BADGE and BFDGE in canned energy drinks by molecularly imprinted polymer cleaning up and UPLC with fluorescence detection, Food Chem. 220 (2017) 406-412, doi:10.1016/j.foodchem.2016. 10.005 .

[2] G. Russo, F. Barbato, L. Grumetto, Development and validation of a LC-FD method for the simultaneous determination of eight bisphenols in soft drinks, Food Anal. Meth. 9 (10) (2016) 2732-2740, doi:10.1007/s12161-016-0458-X.

[3] T. Geens, T.Z. Apelbaum, L. Goeyens, H. Neels, A. Covaci, Intake of bisphenol A from canned beverages and foods on the Belgian market, Food Addit. Contam. 27 (11) (2010) 1627-1637, doi:10.1080/19440049.2010.508183.

[4] European Commission, Commission Regulation (EC) No. 1895/2005 of 18 November 2005 on the restriction of use of certain epoxy derivatives in materials and articles intended to come into contact with food, J. Eur. Union L 302 (2005) 28-32 Available online: http://data.europa.eu/eli/reg/2005/1895/oj (accessed on 20 June 2020).

[5] EFSA, Opinion of the EFSA panel on food contact materials, enzymes, flavourings and processing aids on the risks to public health related to the presence of bisphenol A (BPA) in foodstuffs: Executive summary, EFSA J 13 (2015) 3978, doi:10.2903/j.efsa.2015.3978.

[6] European Commission, Commission Regulation (EU) No. 2018/213, on 12 February 2018 on the use of bisphenol A in varnishes and coatings intended to come into contact with food, off. J. Eur. Union L41 (2018) 6-12 Available online: https://eur-lex.europa.eu/eli/reg/2018/213/oj . (accessed on 20 June 2020).

[7] European Commission, Commission Regulation (EU) No. 10/2011, on plastic materials and articles intended to come into contact with food, Off. J. Eur. Union 12 (2011) 1-89 Available online: http://data.europa.eu/eli/reg/2011/10/oj . (accessed on 20 June 2020).

[8] Y. Yang, J. Yu, J. Yin, B. Shao, J. Zhang, Molecularly imprinted solid-phase extraction for selective extraction of bisphenol analogues in beverages and canned food, J. Agric. Food Chem. 62 (46) (2014) 11130-11137, doi:10.1021/ jf5037933.

[9] J.I. Cacho, N. Campillo, P. Viñas, M. Hernández-Córdoba, Stir bar sorptive extraction coupled to gas chromatography-mass spectrometry for the determination of bisphenols in canned beverages and filling liquids of canned vegetables, J. Chromatogr. A 1247 (2012) 146-153, doi:10.1016/j.chroma.2012.05.064.

[10] S.C. Cunha, C. Almeida, E. Mendes, J.O. Fernandes, Simultaneous determination of bisphenol A and bisphenol B in beverages and powdered infant formula by dispersive liquid-liquid micro-extraction and heart-cutting multidimensional gas chromatography-mass spectrometry, Food Addit. Contam., Part A 28 (2011) 513-526, doi:10.1080/19440049.2010.542551.

[11] H. Gallart-Ayala, E. Moyano, M.T. Galceran, Analysis of bisphenols in soft drinks by on-line solid phase extraction fast liquid chromatography-tandem mass spectrometry, Anal. Chim. Acta 683 (2011) 227-233, doi:10.1016/j.aca.2010.10. 034.

[12] GEMS-Food Euro, Report on a workshop in the frame of GEMS-Food Euro, EUR/HFA target 22, in: Proceedings of the Second Workshop on Reliable Evaluation of Low-Level Contamination of Food, Kulmbach, Germany, 26-27 May, 1995, pp. 26-27.

[13] A. Lestido Cardama, R. Sendón, J. Bustos, M.I. Santillana, P. Paseiro Losada, A. Rodríguez Bernaldo de Quirós, GC-MS screening for the identification of potential migrants present in polymeric coatings of food cans, Polymers 11 (12) (2019) 2086, doi:10.3390/polym11122086.

[14] R. Sendón García, P. Paseiro Losada, C. Pérez Lamela, Determination of compounds from epoxy resins in food simulants by HPLC-fluorescence, Chromatographia 58 (2003) 337-342, doi:10.1365/s10337-003-0048-0.

[15] B. Geueke, FPF Dossier: Can Coatings, Food Packaging Forum, Zurich, Switzerland, 2016, doi:10.5281/zenodo.200633.

[16] M.A. Mekewi, A.M. Ramadan, F.M. ElDarse, M.H.A. Rehim, N.A. Mosa, M.A Ibrahim, Preparation and characterization of polyurethane plasticizer for flexible packaging applications: natural oils affirmed access, Egypt. J. Pet. 26 (1) (2017) 9-15, doi:10.1016/j.ejpe.2016.02.002.

[17] M. Biedermann, K. Grob, Phenolic resins for can coatings: I. Phenol-based resole analysed by GC-MS, GC $\times$ GC, NPLC-GC and SEC, LWT-Food Sci. Technol. 39 (6) (2006) 633-646, doi:10.1016/j.lwt.2005.04.008.

[18] A.K. Mukhopadhyay, Industrial Chemical Cresols and Downstream Derivatives. Eds, CRC press, Boca Ratón, Florida, 2004 ISBN: 978-0-203-99741-3.

[19] A.C. De Groot, M.A. Flyvholm, G. Lensen, T. Menné, P.J. Coenraads, Formaldehyde-releasers: relationship to formaldehyde contact allergy. Contact allergy to formaldehyde and inventory of formaldehyde-releasers, Contact Dermatitis 61 (2) (2009) 63-85, doi:10.1111/j.1600-0536.2009.01582.x.

[20] K. Aalto-Korte, M. Pesonen, K. Suuronen, Occupational allergic contact dermatitis caused by epoxy chemicals: occupations, sensitizing products, and diagnosis, Contact Dermatitis 73 (6) (2015) 336-342, doi:10.1111/cod.12445.
[21] A. Schaefer, V.A. Ohm, T.J. Simat, Migration from can coatings: Part 2. Identification and quantification of migrating cyclic oligoesters below $1000 \mathrm{Da}$, Food Addit. Contam. 21 (4) (2004) 377-389, doi:10.1080/02652030310001637939.

[22] E. Löser, G. Stropp, Polymers, in: Toxicology, Academic Press, 1999, pp. 919936, doi:10.1016/B978-012473270-4/50097-3.

[23] OECD SIDS. (2004). Initial Assessment Report for SIAM 19 ( $\varepsilon$-Caprolactone), 184.

[24] M.A. Lago, L.K. Ackerman, Identification of print-related contaminants in food packaging, Food Addit. Contam. Part A 33 (3) (2016) 518-529, doi:10.1080/ 19440049.2015.1136435.

[25] C. Nerín, P. Alfaro, M. Aznar, C. Domeño, The challenge of identifying nonintentionally added substances from food packaging materials: a review, Anal. Chim. Acta 775 (2013) 14-24, doi:10.1016/j.aca.2013.02.028.

[26] C. Domeño, M. Aznar, C. Nerín, F. Isella, M. Fedeli, O. Bosetti, Safety by design of printed multilayer materials intended for food packaging, Food Addit. Contam. Part A 34 (7) (2017) 1239-1250, doi:10.1080/19440049.2017.1322221.

[27] S.M. Magami, Functional can coatings Part 2: Composition, attributes, applications and performance, Surf. Coat. Int. 96 (3) (2013) 148-155.

[28] K.J. Groh, T. Backhaus, B. Carney-Almroth, B. Geueke, P.A. Inostroza, A. Lennquist, H.A. Leslie, M. Maffini, D. Slunge, L. Trasande, A.M. Warhurst, J. Muncke, Overview of known plastic packaging-associated chemicals and their hazards, Sci. Total Environ. 651 (2019) 3253-3268, doi:10.1016/j.scitotenv. 2018.10.015.

[29] M.R. Thomas, Updated by Staff, Salicylic acid and related compounds, Kirk-Othmer Encycl. Chem. Technol. (2006), doi:10.1002/0471238961. 1901120920081513.a01.pub2.

[30] I. Skjevrak, C. Brede, I.L. Steffensen, A. Mikalsen, J. Alexander, P. Fjeldal, H. Herikstad, Non-targeted multi-component analytical surveillance of plastic food contact materials: Identification of substances not included in EU positive lists and their risk assessment, Food Addit. Contam. 22 (10) (2005) 1012-1022, doi:10.1080/02652030500090877.

[31] I. Clemente, M. Aznar, C. Nerín, O. Bosetti, Migration from printing inks in multilayer food packaging materials by GC-MS analysis and pattern recognition with chemometrics, Food Addit. Contam. Part A 33 (4) (2016) 703-714, doi:10.1080/19440049.2016.1155757.

[32] A. Guart, M. Wagner, A. Mezquida, S. Lacorte, J. Oehlmann, A. Borrell, Migration of plasticisers from TritanTM and polycarbonate bottles and toxicological evaluation, Food Chem. 141 (1) (2013) 373-380, doi:10.1016/j.foodchem.2013. 02.129.

[33] ECHA1-butoxypropan-2-ol, European Chemical Agency, 2019 Accessed on 2020 https://echa.europa.eu/es/substance-information/-/substanceinfo/100.023.526

[34] M.V. Russo, I. Notardonato, P. Avino, G. Cinelli, Fast determination of phthalate ester residues in soft drinks and light alcoholic beverages by ultrasound/vortex assisted dispersive liquid-liquid microextraction followed by gas chromatography-ion trap mass spectrometry, RSC Adv. 4 (103) (2014) 5965559663, doi:10.1039/C4RA08574D.

[35] D.L. Trumbo, C.L. Giddings, L.R.A. Wilson, Terpene-anhydride resins as coating materials, J. Appl. Polym. Sci. 58 (1) (1995) 69-76, doi:10.1002/app.1995. 070580107.

[36] C. Nerín, C. Rubio, J. Cacho, J. Salafranca, Parts-per-trillion determination of styrene in yoghurt by purge-and-trap gas chromatography with mass spectrometry detection, Food Addit. Contam. 15 (3) (1998) 346-354, doi:10.1080/ 02652039809374650.

[37] C. Nerín, D. Acosta, C. Rubio, Potential migration release of volatile compounds from plastic containers destined for food use in microwave ovens, Food Addit. Contam. 19 (6) (2002) 594-601, doi:10.1080/02652030210123887.

[38] C. Dutra, D. Pezo, M.T. de Alvarenga Freire, C. Nerín, F.G.R. Reyes, Determination of volatile organic compounds in recycled polyethylene terephthalate and high-density polyethylene by headspace solid phase microextraction gas chromatography mass spectrometry to evaluate the efficiency of recycling processes, J. Chromatogr. A 1218 (10) (2011) 1319-1330, doi:10.1016/j.chroma.2010. 12.099.

[39] E.L. Bradley, M. Driffield, N. Harmer, P.K.T. Oldring, L Castle, Identification of potential migrants in epoxy phenolic can coatings, Int. J. Polym. Anal. Charact. 13 (3) (2008) 200-223, doi:10.1080/10236660802070512.

[40] Y. Chang, C. Nguyen, V.R. Paranjpe, F. Gilliland, J.J. Zhang, Analysis of bisphenol A diglycidyl ether (BADGE) and its hydrolytic metabolites in biological specimens by high-performance liquid chromatography and tandem mass spectrometry, J. Chromatogr. B 965 (2014) 33-38, doi:10.1016/j.jchromb.2014.06. 005.

[41] I. Clemente, M. Aznar, C. Nerín, O. Bosetti, Migration from printing inks in multilayer food packaging materials by GC-MS analysis and pattern recognition with chemometrics, Food Addit. Contam. Part. A 33 (2016) 703-714, doi:10.1080/19440049.2016.1155757.

[42] M.N.H. Rozaini, N. Yahaya, B. Saad, S. Kamaruzaman, N.S.M Hanapi, Rapid ultrasound assisted emulsification micro-solid phase extraction based on molecularly imprinted polymer for HPLC-DAD determination of bisphenol A in aqueous matrices, Talanta 171 (2017) 242-249, doi:10.1016/j.talanta.2017.05.006.

[43] N. González, S.C. Cunha, R. Ferreira, J.O. Fernandes, M. Marquès, M. Nadal, J.L. Domingo, Concentrations of nine bisphenol analogues in food purchased from Catalonia (Spain): Comparison of canned and non-canned foodstuffs, Food Chem. Toxicol. 136 (2020) 110992, doi:10.1016/j.fct.2019.110992.

[44] S. Biedermann, M. Zurfluh, K. Grob, A. Vedani, B.J. Brüschweiler, Migration of cyclo-diBA from coatings into canned food: method of analysis, concentration determined in a survey and in silico hazard profiling, Food Chem. Toxicol. 58 (2013) 107-115, doi:10.1016/j.fct.2013.04.004. 